INDO GLOBAL JOURNAL OF

PHARMACEUTICAL SCIENCES

ISSN 2249- 1023

\title{
Novel PGPR Consortium for Confrontation of a Common and Widespread Disease of Paddy Field
}

\author{
Rishi Kumar Verma, Manish Sachan, Shivesh Sharma ${ }^{*}$ \\ Department of Biotechnology, MNNIT Allahabad, Allahabad (UP) 211004, India \\ Address for Correspondance: Shivesh Sharma, shiveshs@mnnit.ac.in
}

Keywords PGPR; Consortia; Rice Disease; Paddy Field.

\begin{abstract}
Five plant growth promotion rhizobacteria (PGPR) consortia have been evaluated for induced systemic resistance against a common and widespread disease of paddy field observed during routine visit to fields. The identification of colonies grown on nutrient agar media is under process and sent for $16 \mathrm{~S}$ rRNA sequencing. Seeds of Oryza sativa var. Samba mahsuri have been treated with consortia suspension (SR-2, SR-6, SR-18, SR-61 and SR-64) and green house trial has been set up to see their effects on growth promotion and disease resistance. Average germination of $86 \%$ was recorded with seeds treated with consortia in comparison with $72 \%$ germination in the untreated control. Pathogens were isolated carefully from leaf samples collected from paddy field and converted into suspension through standard lab protocols. Grown plants were challenged with the isolated one under greenhouse conditions and observed over long time period. We found that these particular bacterial consortia are good growth promoters and able to induce systemic resistance in rice plants. The results of the present study suggest that these consortia used as suspension may have commercial potential. It is evident that rhizobacteria could possibly serve as eco-friendly and sustainable alternatives to the hazardous chemicals used for growth promotion and management of crop diseases. (C) 2016 iGlobal Research and Publishing Foundation. All rights reserved.
\end{abstract}

Conference Proceedings: International Conference on Advances in Plant and Microbial Biotechnology (PMB2017); JIIT, Noida: February 02-04, 2017

Indo Global Journal of Pharmaceutical Sciences( ISSN 22491023 ; CODEN- IGJPAI; NLM ID: 101610675) indexed and abstracted in EMBASE(Elsevier), SCIRUS(Elsevier),CABI, CAB Abstracts, Chemical Abstract Services(CAS), American Chemical Society(ACS), Index Copernicus, EBSCO, DOAJ, Google Scholar and many more. For further details, visit http://iglobaljournal.com 